\title{
Novel transcription regulatory sequences and factors of the immune evasion protein ICP47 (US12) of herpes simplex viruses
}

Jun-Ting Cheng ${ }^{1,2+}$, Ying-Ying Wang ${ }^{1,2+}$, Lin-Zhong Zhu ${ }^{3+}$, Ying Zhang ${ }^{1,2}$, Wen-Qi Cai ${ }^{1,2}$, Zi-Wen Han ${ }^{1,2}$, Yang Zhou ${ }^{1,2}$, Xian-Wang Wang ${ }^{1,4}$, Xiao-Chun Peng ${ }^{1,5}$, Ying Xiang ${ }^{1,2}$, Hui-Yu Yang ${ }^{6}$, Shu-Zhong Cui', Zhaowu Ma ${ }^{1,2^{*}}$, Bing-Rong Liu ${ }^{6^{*}}$ and Hong-Wu Xin ${ }^{1,2,8^{*}}$

\begin{abstract}
Background: Herpes simplex virus (HSV) can cause encephalitis. Its infected cell polypeptide 47 (ICP47), encoded by immediate-early gene US12, promotes immune escape. ICP47 was modified in the clinically approved oncolytic HSV (oHSV) T-Vec. However, transcription regulatory sequence (TRS) and transcription regulatory factor (TRF) of HSV US12 are seldom reported.
\end{abstract}

Methods: Previously, our laboratory isolated a new HSV strain named HSV-1-LXMW from a male patient with oral herpes in Beijing, China. Firstly, the genetic tree was used to analyze its genetic relationship. The US12 TRS and TRF in HSV-1-LXMW were found by using predictive software. Secondly, the further verification by the multi-sequence comparative analysis shown that the upstream DNA sequence of HSV US12 gene contained the conserved region. Finally, the results of literature search shown that the expression of transcription factors was related to the tissue affinity of HSV-1 and HSV-2, so as to increase the new understanding of the transcriptional regulation of HSV biology and oncolytic virus (OVs) therapy.

Results: Here we reported the transcriptional regulation region sequence of our new HSV-1-LXMW, and its close relationship with HSV-1-CR38 and HSV-1-17. Importantly we identified eight different kinds of novel TRSs and TRFs of HSV US12 for the first time, and found they are conserved among HSV-1 (c-Rel, Elk-1, Pax-4), HSV-2 (Oct-1, CF2-II, E74A, StuAp) or both HSVs (HNF-4). The TRFs c-Rel and Oct-1 are biologically functional respectively in immune escape and viral replication during HSV infection.

Conclusions: Our findings have important implication to HSV biology, infection, immunity and oHSVs.

Keywords: HSV-1, HSV-2, US12, ICP47, Transcriptional regulation sequence (TRS), Transcriptional regulation factor (TRF)

\footnotetext{
*Correspondence: zhaowu823@126.com; bingrongliu@qq.com;

hongwu_xin@126.com

†Jun-Ting Cheng, Ying-Ying Wang and Lin-Zhong Zhu contributed equally to

this work.

'Laboratory of Oncology, School of Basic Medicine, Health Science Center, Yangtze University, 1 Nanhuan Road, Jingzhou 434023, Hubei, China

${ }^{6}$ Department of Gastroenterology, The First Affiliated Hospital of Zhengzhou University, Zhengzhou 450052, China

Full list of author information is available at the end of the article
}

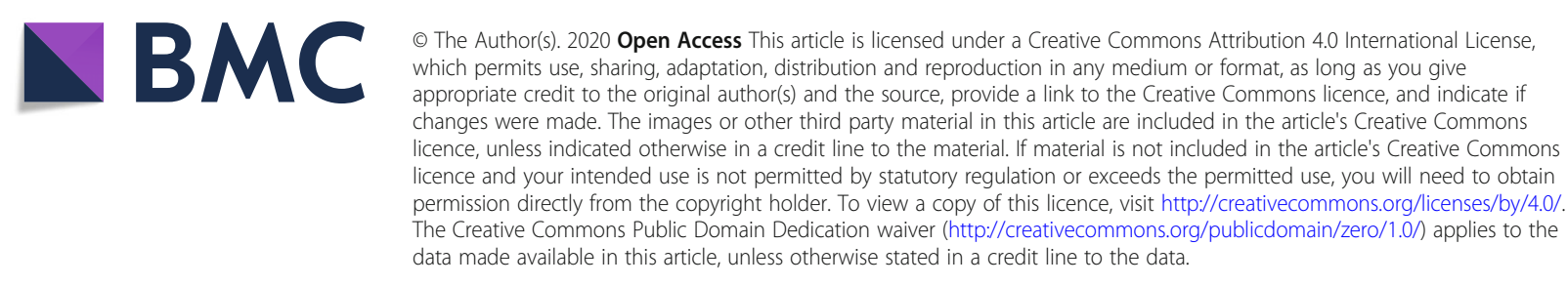




\section{Background}

Tumors are heterogeneous and often resistant to chemotherapy and radiotherapy $[1,2]$, and no single treatment could be widespread applied or has full effectivity for cancer treatment [3-6]. OVs treatment are different from conventional chemotherapy and radiotherapy, and could provide additional treatment strategies [7]. Additionally, OVs are diverse in structure and biology, which spread among tumors with different kinetics and kill tumor cells through multiple mechanisms [8]. The oHSV T-VEC has been approved by FDA for patients with melanoma [911]. HSV, a member of the alpha-herpesviruses subfamily, which is an encapsulated DNA virus, offers particular benefits for use as a gene transfer vector, contains at least 120 $\mathrm{kb}$ of double-stranded DNA genome, encoding more than 70 genes [7, 12]. Type 1 and 2 HSVs (HSV-1 and HSV-2) are the most common and acute human pathogens. HSV1 is normally related to oral-facial infections and may cause encephalitis in severe cases, while HSV-2 mostly induces genital infections and could cause mother-to-child transmission $[13,14]$.

HSV as an OVs has many favourable properties. Engineered oHSVs have been shown to be remarkably safe in clinical trials, and also have some evidence of their effectiveness [12]. Dlsptk, the first recombinant HSV, was generated by deleting the gene UL23 encoding thymidine kinase (TK) [15]. The selectivity and efficacy of dlsptk established a principled proof for the application of HSV-1 genome deletions to carry out the tumor selectivity. However, from the standpoint of clinical application, the UL23 deletion was eventually problematic for it causes dlsptk impervious to first-line anti-herpes pharmaceuticals, resulting in abandance of dlsptk. The second recombinant HSV, G207, is the first oHSV tested in clinical trials and it deletes the genes UL39 (ICP6) and RL1 (ICP34.5). As is well-known, ICP34.5, a key factor of HSV neurovirulence, can preclude the shut-off of protein synthesis in infected host cells. ICP6 is a determinant viral enzyme for HSV DNA synthesis, which is indispensable for virus replication in normal nondividing cells [16]. Dlsptk and G207 are designed to weaken viral replication and reduce viral virulence in non-cancer cells. The third generation oHSV-1 vector, G47 $\Delta$, is based on G207 with additional ICP47 deletion, which surprisingly enhances viral replication and increases immune recognition of infected cells [17]. ICP47 deletion contains the promoter region of US11 and also attenuates $\gamma 34.5$ growth [18]. Importantly, because of ICP47 can block peptide loading of major histocompatibility complex I (MHC-I) molecules, G47 $\Delta$ has induced an antitumor immune response for the ICP47 deletion. Therefore, increasing MHC I antigen presentation, stimulating cytotoxic lymphocytes and reducing NK cytolysis of infected cells can enhance anti-tumor immune response. Anti-tumor immune responses may be the key for the treatment of tumor metastasis .

ICP47, encoded by gene US12, is a polymorphous protein and could block RNA splicing in early infection, and then, shuttle viral mRNA from nucleus to cytoplasm in late infection [19]. ICP47 directly binds antigendependent transporter (TAP), limiting antigen trafficing, leading to the occurrence of empty MHC-I [20]. The functional domain of ICP47 has been mapped to 35 residues at the $\mathrm{N}$-terminal, forming an extended helix-loophelix structure in the lipid bilayer [20]. In addition, since ICP47 is too large to be easily transported by TAP, its high affinity binding traps TAP in an inactive conformation [21]. The binding of ICP47 stabilizes the inward conformation, and therefore blocks TAP from transitioning to the outward state in which the nucleotide binding domains (NBDs) form a closed dimer and the translocation pathway points to the endoplasmic reticulum (ER) cavity [22]. By blocking the entry of viral antigens into ER, HSV could avoid the attrack of cytotoxic $\mathrm{T}$ lymphocytes (CTLs), which may lead to immune escape of HSV and establish lifelong infection in the host cells. Interestingly, the ICP47 in G47 $\Delta$ is deleted, and this keeps the cell surface MHC-I-antigen expression and allows to enhance antigen presentation [18]. Furthermore, G47 $\Delta$ has been proved effective in animal tumor models of various cancers such as brain cancer, prostate cancer, breast cancer, schwannoma and human melanoma [23-25].

Currently, HSV US12 is widely used in OVs modification, gene therapy and vaccine construction [25-27]. However, there are no reports on TRS and TRF of US12 gene in HSV. As an immediate-early protein, its expression is regulated by the tri-partite Oct-1/HCF/VP16 complex [28, 29]. Identification of additional conserved promoter regulatory sequences that might further regulate its expression is certainly an important question. Here we sequenced the transcriptional regulation region of US12 of our new HSV-1 strain LXMW, and for the first time identified novel TRS and TRF of HSV US12. These findings may have important impactions for HSV biology, infection, immunity and OVs.

\section{Methods}

Previously, our laboratory isolated a new HSV strain named HSV-1-LXMW from a male patient with oral herpes in Beijing, China [30]. The detailed content of Cells, HSV-1 isolation and identification, and HSV genomic DNA sequencing analysis have been elaborated [30].

\section{Identification of the US12 potential transcriptional regulation region sequences in $\mathrm{HSV}$}

The online program NCBI (National Center for Biotechnology Information: https://www.ncbi.nlm.nih.gov/) was 
used to determine US12 potential transcriptional regulation region sequences.

\section{Phylogenetic analysis of the transcriptional regulation region of the US12 gene}

We used MEGA7 (https://www.megasoftware.net/), the application (APP), to analyze phylogenetic relationship.

\section{Prediction of US12 transcription regulatory sequences and factors}

We used online program Match (http://gene-regulation. com/pub/programs.html) to predict the gene US12 TRS and TRF according to their instruction.

\section{Alignment of the transcriptional regulation region sequences of US12}

ApE (A plasmid Editor: http://biologylabs.utah.edu/jorgensen/wayned/ape/), the application (APP), was used to make the potential transcriptional regulation region sequences alignment of gene US12 according to their manual.

\section{Results}

Identification of the US12 transcription regulatory region of HSV-1-LXMW

Using the nucleotide sequence database, we identified transcription regulatory regions of US12 (145851-148, 050) as shown in Fig. 1. Please refer to supplementary material for the sequence of the transcription regulatory region. The transcription regulatory regions are $2000 \mathrm{bp}$ upstream and $200 \mathrm{bp}$ downstream of US12 transcription initiation sites. Interestingly, the gene that encodes ICP47 is US10, not US12 in HSV-2 strain HG52 and H1226. We summarized information about the HSV US12 genomic DNA transcription regulatory regions of our new strain and 11 other strains studied in this article (Table 1).
Phylogenetic analysis of HSV-1-LXMW and other 11 HSV strains

Based on the gene US12 transcription regulatory region sequences in Table 1 of HSV-1-LXMW and other 11 HSV strains, including 8 HSV-1 strains (17, CR38, H129, SC16, KOS, Patton, E19 and F) and 3 HSV-2 strains (SD90e, HG52 and H1226), the phylogenetic analysis about the evolutionary relationship among HSV-1LXMW and other $11 \mathrm{HSV}$ strains were performed. The result shown high homology among our new strain HSV-1-LXMW and strains HSV-1-CR38, HSV-1-17 and HSV-1-H129. Our data again support that HSV-1LXMW is a strain of HSV-1 (Fig. 2).

\section{Identification of the US12 TRS and TRF}

Better understanding of $U S 12$ transcriptional regulation is crucial for HSV biology and antitumor immune responses of oHSVs. Using Match, the online program, we find four major TRS of HSV-1-LXMW, which bind to c-Rel, HNF4, ElK-1 and Pax-4, and three of HSV-1-17, which bind to c-Rel, HNF-4 and Pax-4. Interestingly, compared with the TRF of HSV-2, the difference between HSV-1 and HSV-2 is quite large. We find five major different kinds of TRSs of HSV-2-SD90e, which bind to HNF-4, CF2-II, E74A, Oct-1 and StuAp (Table 2).

Further analysis of three more HSV-1 strains found that their TRS and TRF binding sites are similar with HSV-1-LXMW, but have minor differences (Fig. 3). Comparing to HSV-1-LXMW, there is no c-Rel binding site for HSV-1-SC16, no Elk-1 binding site for HSV-1Patton and 17, and no Pax-4 binding site for HSV-1Patton and E19. There is only one US12 TRS binding to HNF-4 in all the HSV-1 strains analyzed, but there are two HNF-4 binding sites in HSV-2 strains.

\section{The TRS and TRF are conserved}

Conserved sequences refer to highly similar or identical nucleic acid sequences (RNA or DNA sequences),

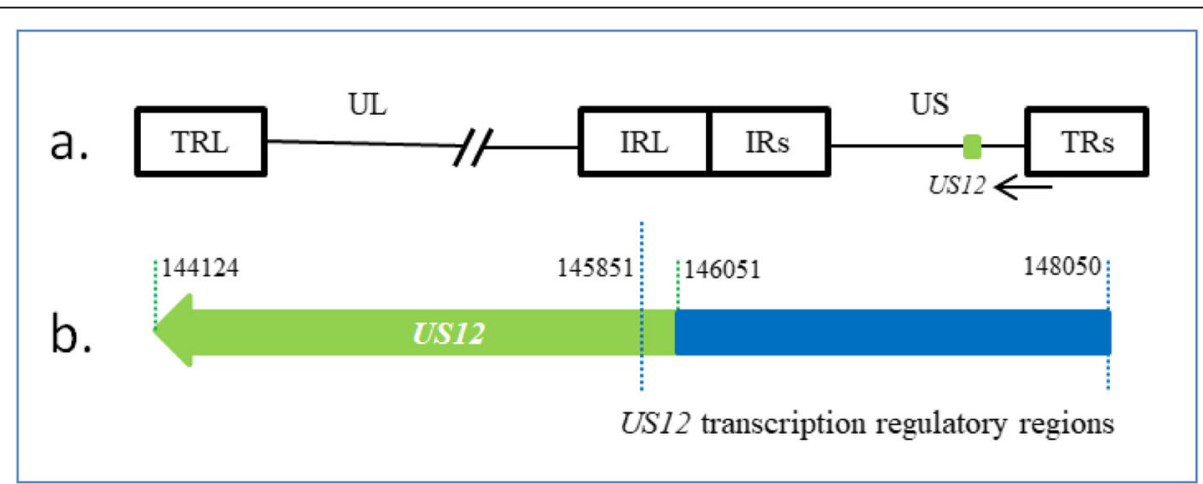

Fig. 1 Transcription regulatory regions of US12. a. Schematic of the HSV-1 genome showing the regions of US12. The HSV-1 genome consists of long and short unique regions (UL and US) each bounded by terminal (T) and internal (I) repeat regions (RL and RS). b. The DNA sequence of the US12 gene is marked in green and the transcription regulatory regions of US12 is marked in blue 
Table 1 HSV US12 genomic DNA sequencing

\begin{tabular}{llllll}
\hline HSV Strain & Gene Bank ID & Tax-ID & Sub-Date & US12 transcription regulatory regions & University, Country \\
\hline HSV-1 strain XLMW & & & & $145,851 / 148,050$ & Yangtze University, Jingzhou, China \\
HSV-1 strain 17 & JN555585 & 10,299 & $2011-08-02$ & $145,869 / 148,068$ & RC University, Glasgow, UK \\
HSV-1 strain H129 & GU734772 & 744,249 & $2010-01-18$ & $145,769 / 147,968$ & Princeton University, USA \\
HSV-1 Strain CR38 & HM585508 & 10,298 & $2013-10-17$ & $145,315 / 147,514$ & MRC Virology Unit, UK \\
HSV-1 strain SC16 & KX946970 & 10,309 & $2016-10-30$ & $113,629 / 114,168$ & Severo Ochoa, Spain \\
HSV-1 strain KOS & JQ673480 & 10,306 & $2012-03-10$ & $145,672 / 147,871$ & University of Kansas, USA \\
HSV-1 strain Patton & MF959544 & 10,308 & $2017-10-11$ & $146,470 / 148,669$ & NYU, New York, USA \\
isolate & & & & & University of Glasgow Centre for Virus \\
HSV-1 strain E19 & HM585511 & 10,298 & $2013-10-22$ & $144,988 / 147,187$ & Research, UK \\
& & & & & Princeton University, USA \\
HSV-1 strain F & GU734771 & 10,304 & $2010-01-18$ & $145,795 / 147,994$ & Harvard Medical School, Boston, US \\
HSV-2 strain SD90e & KF781518 & 1,177, & $2013-10-25$ & $147,503 / 149,702$ & University of Glasgow, UK \\
HSV-2 strain HG52 & JN561323 & 10,310 & $2011-08-05$ & $147,834 / 150,033$ & Pennslyvania State University, USA \\
HSV-2 strain H1226 & KY922720 & 16,866 & $2017-09-27$ & $147,094 / 149,293$ &
\end{tabular}

protein sequences, and their structures. Conserved sequences generally have functional value. Here, we found that the US12 TRSs and TRF binding sites are conserved among the 9 HSV-1 and 3 HSV-2 strains (Fig. 4), indicating these conserved TRSs and TRFs are likely to be biologically functional.

Our multi-sequence alignment results indicated that in the US12 transcriptional regulation regions there are less mutations among HSV-1 strains than mutations between HSV-1 and HSV-2. Between HSV-1-LXMW and HSV$1-172,184$ base pairs are matched and only 5 base pairs are mismatched. However, there are only 468 base pairs matched between the HSV-1- LXMW and HSV-2SD90e. Therefore, we decided to compare HSV type 1 and type 2 separately in ApE Program (Aligment parameters: Blocks: 10, mismatch penalty: 0, gap penalty: 0, gap Ext penalty: 0, everything else is at default). Results shown that our new strain HSV-1-LXMW was highly similar to HSV-1-17 and HSV-1-CR38 (Fig. 4a).

The alignment of the transcription regulatory region (nucleotides 1-2200) of HSV-1-XLMW strain to other 8 HSV-1 strains SC16, Patton, KOS, H129, F, E19, CR38 and 17, respectively, shown 2177, 2023, 2182, 2165, 2167, 2150, 2184 and 2184 matched base pairs, 11, 6, 6,

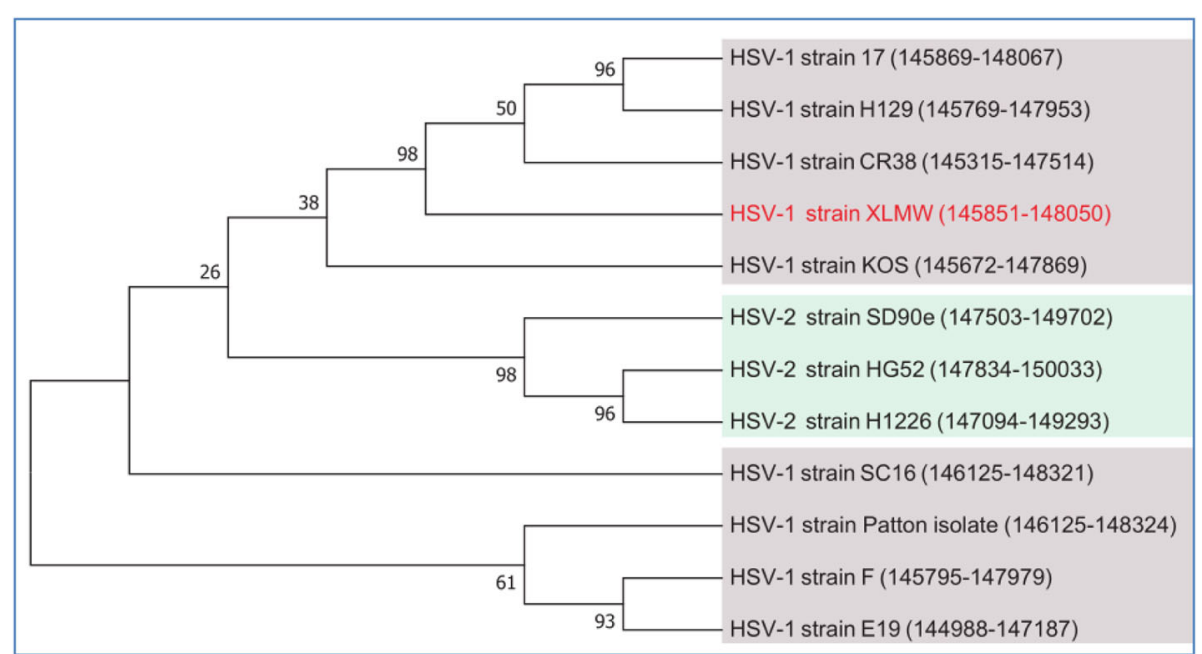

Fig. 2 Phylogenetic analysis of HSV-1-LXMW together with 11 other HSV strains. Evolutionary analyses were conducted in MEGA7. The evolutionary history was inferred by using the Maximum Likelihood method based on the Tamura-Nei model. The bootstrap consensus tree inferred from 1000 replicates is taken to represent the evolutionary history of the taxa analyzed. Branches corresponding to partitions reproduced in less than $50 \%$ bootstrap replicates are collapsed 
Table 2 The US12 TRS and TRF in HSVS

\begin{tabular}{|c|c|c|c|c|c|c|}
\hline HSV strain & Matrix identifier & Position strand & Core match & Matrix match & Sequence & Factor name \\
\hline \multirow[t]{4}{*}{ HSV-1 strain XLMW } & V\$CREL_01 & $37(+)$ & 1.000 & 0.982 & gggtcTTCC & c-Rel \\
\hline & V\$HNF4_01 & $1020(-)$ & 0.883 & 0.898 & ccctgtcCTTTTtcccacc & HNF-4 \\
\hline & V\$ELK1_02 & $1875(+)$ & 1.000 & 0.984 & ggcgcCGGAAgccc & Elk-1 \\
\hline & V\$PAX4_01 & $2029(-)$ & 0.888 & 0.833 & gccacgggecgCTTCAcggcc & Pax-4 \\
\hline \multirow[t]{3}{*}{ HSV-1 strain 17} & V\$CREL_01 & $37(+)$ & 1.000 & 0.982 & gggtcTाCC & c-Rel \\
\hline & V\$HNF4_01 & $1023(-)$ & 0.883 & 0.898 & ccctgtcCTTTTtcccacc & HNF-4 \\
\hline & V\$PAX4_01 & $2032(-)$ & 0.888 & 0.833 & gccacgggecgCTTCAcggcc & Pax-4 \\
\hline \multirow[t]{7}{*}{ HSV-2 strain SD90e } & V\$HNF4_01 & $746(-)$ & 1.000 & 0.928 & gctcgcaCTTTGccctaat & HNF-4 \\
\hline & |\$CF2||_01 & $767(-)$ & 1.000 & 1.000 & tatATATAc & CF2-II \\
\hline & V\$HNF4_01 & $886(-)$ & 1.000 & 0.928 & gctcgcaCTTTGccctaat & HNF-4 \\
\hline & |\$CF2||_01 & $907(-)$ & 1.000 & 1.000 & tatATATAc & CF2-II \\
\hline & |\$E74A_01 & $1076(+)$ & 1.000 & 0.954 & cgaaccCGGAAgggcag & E74A \\
\hline & V\$OCT1_Q6 & $1120(-)$ & 0.883 & 0.911 & ctcaTTAGCatcgcg & Oct-1 \\
\hline & F\$STUAP_01 & $1637(-)$ & 1.000 & 1.000 & ggtCGCGAtg & StuAp \\
\hline
\end{tabular}

$11,11,10,3$ and 5 mismatched base pairs, and 24, 324, 26, 48, 44, 80, 26 and 21 base pair gaps (Fig. 4a). The alignment of the transcription regulatory region (nucleotides 1-2200) of HSV-2- SD90e strain to other $2 \mathrm{HSV}-2$ strains HG52 and H1226, respectively, shown 1956 and 2128 matched base pairs, 3 and 2 mismatched base pairs, and 480 and 138 base pair gaps (Fig. 4b).

A sequence with five or more conserved base pairs is defined as a conservative region. From the alignment analysis, we found 14 conserved regions of $9 \mathrm{HSV}-1$ strains and 7 conserved regions of 3 HSV-2 strains (Fig. 4). The conserved regions of HSV-1-LXMW US12 started respectively at $1,37,58,70,79,1019,1036,1043,1104$, $1807,1833,1842,1886$, and 2007 base pairs. Interestingly, we found that most of the US12 transcriptional regulation regions are overlapped with the TRSs identified above.

There are four TRFs in HSV-1-LXMW, KOS, H129, F and CR38 strains and three TRFs in HSV-1-SC16, E19

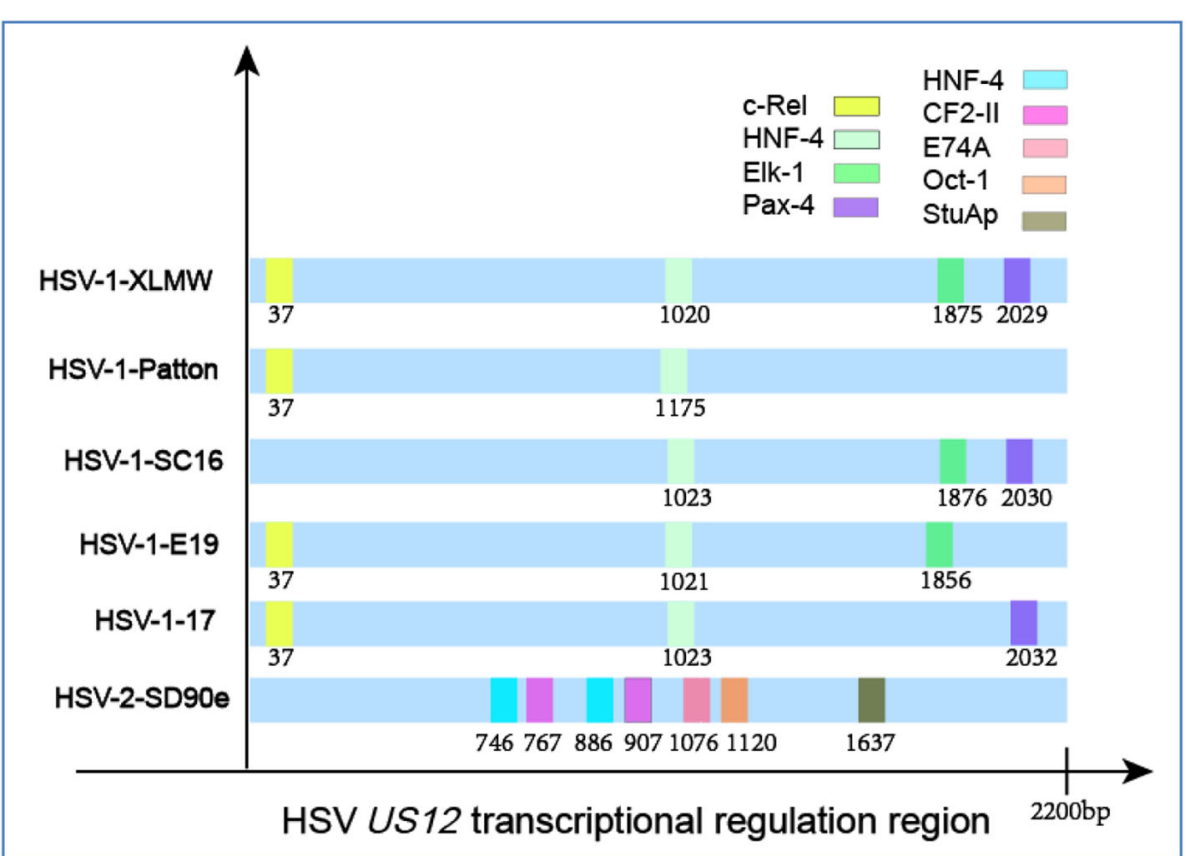

Fig. 3 The US12 TRSS and TRFs in HSVs. TRFs are represented in different colors, and the number represents the specific location of their binding TRFS 


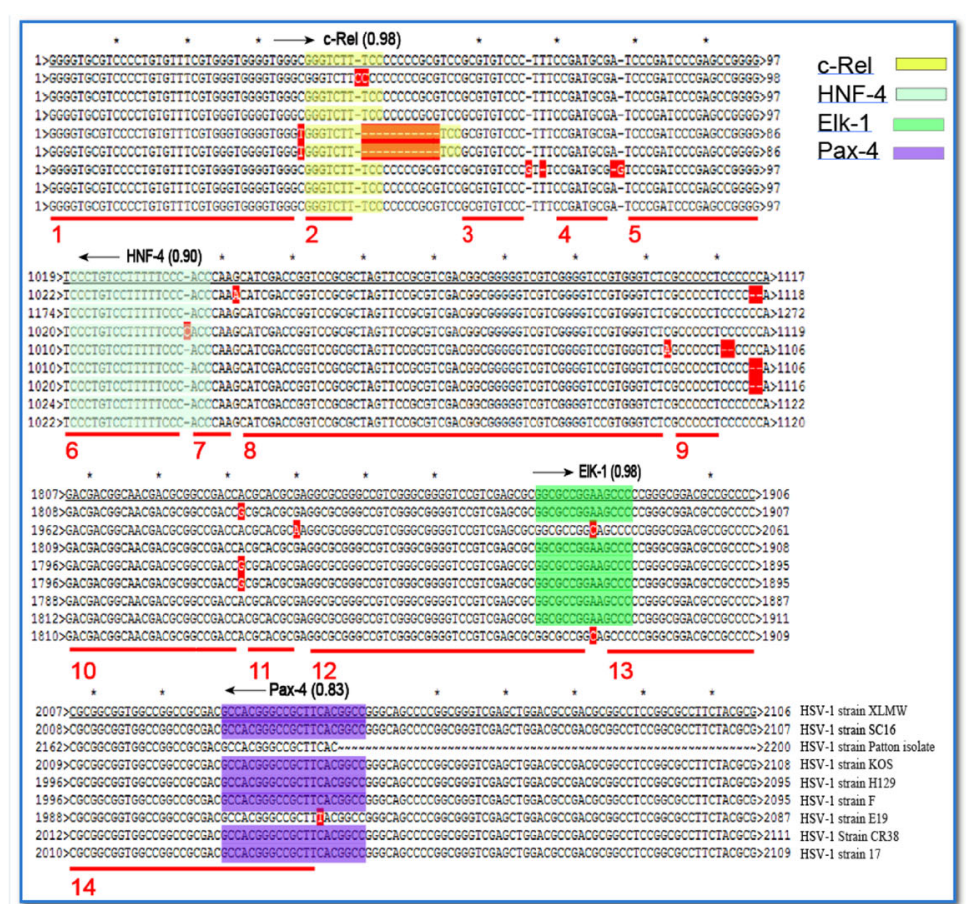

(a).

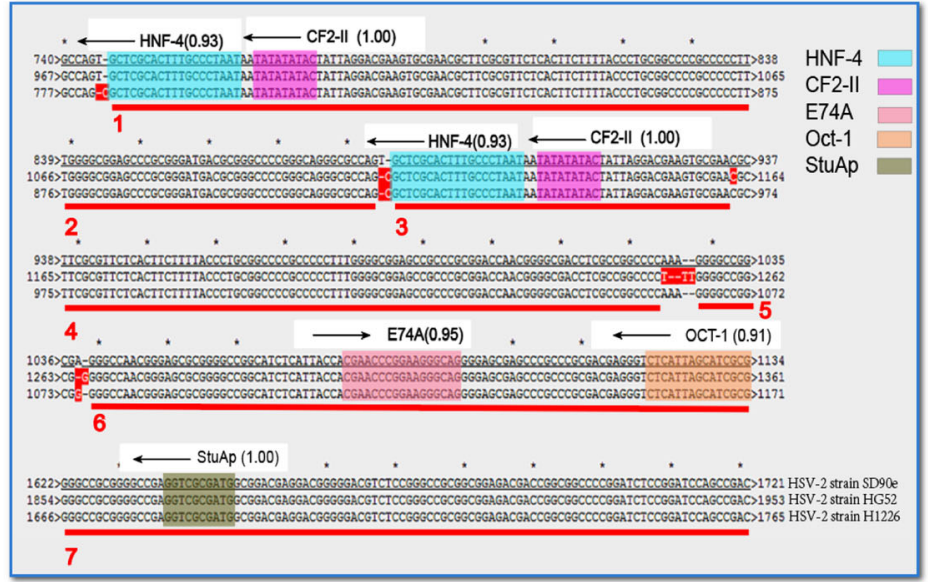

(b).

Fig. 4 a. The US12 TRSS and TRFs are conserved among HSV-1 strains. Red lines indicate the conserved regions 1-14. The TRSS and TRFs are shown in colored boxes. $\mathbf{b}$ The US12 TRSS and TRFs are identical among HSV-2 strains. Red lines indicate the conserved regions 1-17. The TRSS and TRFs are shown in colored boxes

and 17 strains and two transcription factors in HSV1-patton strain. Additionally, the TRFs binding sites of the HSV-1 strains are basically conserved (Fig. 4a). Interestingly, TRSs in the US12 transcriptional regulatory region are identical in $\mathrm{HSV}-2$ strains SD90e, HG52 and H1226 (Fig. 4b). Importantly, binding sites of c-Rel, HNF-4, Elk-1, Pax-4, CF2-II, E74A, Oct-1 or StuAp in US12 transcriptional regulation regions are also conserved among HSV-1/2. HNF-4 is conserved in both HSV-1 and HSV-2 strains. Our findings support that the conserved TRSs and TRFs binding sites are closely linked with the gene US12 functions. However, the TRSs and TRFs between HSV-1 and HSV-2 strains are quite different. Many bases are found unpaired in the sequence alignment, and that indicates different biological functions of these TRSs and TRFs in HSV-1 or HSV-2. Whether their functions exist in vivo or are related to the immune escape of HSV is not clear, thus, to validate these, further functional studies are needed. 


\section{The TRFs c-Rel and Oct-1 are functional during HSV infection}

To understand whether the identified conserved TRSs and TRFs are functional or are involved in the immune escape of HSV, we did a literature search of each of these TRSs and TRFs related to HSV-1 or HSV-2. We found that the TRFs of c-Rel and Oct-1 have been reported to be expressed and functional respectively in HSV-1 and HSV$1 / 2$ infected cells (Table 3 ). These data are consistent with the identification of TRF c-Rel binding site in HSV-1 and Oct-1 binding site in HSV-2 (Fig. 3), supporting that these TRSs and TRFs are biologically functional.

It is well known that HSV-1 causes buccal ulcers and encephalitis. Interestingly, it's reported that c-Rel is a novel cause of herpes simplex encephalitis susceptibility [38]. Studies have also shown that c-Rel is involved in immune evasion via interacting with viral nuclear protein UL24 and endogenous NF-kB subunits p65 and p50, and inhibiting cGAS-STING mediated NF- $\mathrm{kB}$ promoter activity in HSV-1 infected cells. We would hypothesize that our newly identified HSV-1 specific c-Rel may bind to its US12 TRSs, and activate US12 (ICP47) expression in HSV-1 infected cells. In turn, ICP47 blocked HSV-1 antigen presentation, and promoted HSV-1 infection spread and herpes simplex encephalitis. Oct-1 activates IE-gene transcription through forming a transactivation complex with the cellular proteins HCF-1 and VP16 tegument protein in HSV-1 infected tissues [28, 29].

However, there was no report of US12 transcriptional regulation by c-Rel or Oct-1, no report on c-Rel expression in HSV-2 infected tissues, and no report about expression and function of the other 6 identified TRFs of HNF-4, Elk-1, Pax-4, CF2-II, E74A and StuAp, and no report of any of the TRSs identified above in HSV.

\section{The HSV-1/2 tissue tropism and TRFs expression in different tissues}

Tissue tropism is the cells and tissues of a host that support the growth of a particular virus or bacterium. Some bacteria and viruses have a wide range of tissue tropism and can infect many types of cells and tissues, while other viruses may infect mainly individual tissues. Here we summarized the HSV-1/2 tissue tropism and the TRFs expression in different tissues (Table 4). According to the results, HSV-1 specific c-Rel, Elk-1, and Pax-4 are highly expressed in tissues above the abdomen, including oral cavity, tongue and head, and Oct-1, HNF-4 and CF2-II are highly expressed in tissues within the genital system. c-Rel belongs to the nuclear factor $\kappa B(N F-\kappa B)$ family, and plays a crucial role in mammalian $B$ and $T$ cell function [39]. Elk-1 is involved in ERK-induced cellular proliferation, and its transcriptional activity is regulated by ubiquitination at lysine 35 (K35) [40]. Pax proteins are crucial in stem cell biology and organ development. Pax-4 is known to be a major regulator of pancreatic cell development and differentiation, and its transactivation domain was localized within its Cterminal region [41]. OCT-1 (Pou2f1) is well known as a widely expressed TRFs in most cells and tissues. Recently, a series of studies have reported that OCT-1 plays a critical role in $\mathrm{CD}_{4}^{+} \mathrm{T}$ cell function through mediating long-range chromosomal interactions and regulating gene expression during differentiation [42]. Hepatocyte nuclear factor 4 (HNF-4) is enriched in liver extracts and belongs to the steroid hormone receptor superfamily [43]. C(2)-H(2)-type zinc-finger transcription factor II (CF2-II) may potentially regulates diverse sets of target genes during cell development and the CF2-II recognition properties depends largely on the $\mathrm{COOH}$-terminal DNA binding

Table 3 The TRFs c-Rel and Oct-1 are functional during HSV infection

\begin{tabular}{|c|c|c|c|c|c|}
\hline Tissue type & HSV strain & Oct-1 & c-Rel & Function & Ref. \\
\hline Kidney: Vero cells & HSV-1 strain 17 & - & c-Rel & As a novel cause of HSE disease susceptibility. & {$[31]$} \\
\hline $\begin{array}{l}\text { Hematological: Jurkat } \\
\text { cells }\end{array}$ & HSV-1 & - & p65/c-Rel & $\begin{array}{l}\text { the p65/c-Rel heterodimer is responsible for the NF-kB-dependent induction } \\
\text { of HIV-1 LTR in HSV- 1-infected cells. }\end{array}$ & {$[32]$} \\
\hline $\begin{array}{l}\text { Embryonic: WT and } \\
\text { dOct MEF cells }\end{array}$ & HSV-1 strain F & Oct-1 & - & $\begin{array}{l}\text { Oct-1 is required for the formation of HSV replication factories and late gene } \\
\text { expression. }\end{array}$ & [33] \\
\hline Digestive: Hep2 cells & HSV-1 strain KOS & Oct-1 & - & Oct-1 directly recognizes TAATGARAT elements in promoters of IE genes. & [34] \\
\hline Urinary: COS-7 cells & HSV-1 strain KOS & Oct-1 & - & Distinct conformations of Oct-1 on the BHV IE1 sites and on the HSV IE110 sites. & {$[35]$} \\
\hline Genital: HeLa cells & HSV-1 strain F & Oct-1 & - & $\begin{array}{l}\text { late in infection Oct-1 is posttranslationally modified and exhibits a reduced } \\
\text { capacity to bind to its cognate sites. }\end{array}$ & [36] \\
\hline Genital: HeLa cells & HSV-1 strain KOS & Oct-1 & - & $\begin{array}{l}\text { Ser375 is important for the interaction of VP } 16 \text { with Oct-1, and that the interaction } \\
\text { is required to enable both proteins to bind to IE promoters. }\end{array}$ & {$[28]$} \\
\hline Genital: HFF & HSV-1 strain KOS & Oct-1 & - & $\begin{array}{l}\text { forms a transactivation complex with the cellular proteins HCF-1 and HSV-1 VP16 } \\
\text { tegument protein. }\end{array}$ & [29] \\
\hline Genital: HeLa cells & HSV-2 strain 333 & Oct-1 & - & $\begin{array}{l}\text { the HSV-2 protein forms a transcriptional complex with the cellular Oct-1 protein } \\
\text { and target TAATGARAT elements from immediate-early promoters. }\end{array}$ & [37] \\
\hline
\end{tabular}


Table 4 The HSV-1/2 tissue tropism and the TRFs expression in different tissues $H$ high-expression, $M$ middle expression, $L$ little expression, $N$ no-expression; The result from: http://biogps.org. Grading was based on fold increases compared to median fluorescence intensity on Affymetrix microarray chips at 0-2.5 (L), $>2.5-<5(\mathrm{M}),>5(\mathrm{H})$

\begin{tabular}{|c|c|c|c|c|c|c|c|c|c|}
\hline System & Cell/ tissue & HSV-1 & HSV-2 & c-Rel & HNF-4 & Elk-1 & Pax-4 & CF2-II & OCT-1 \\
\hline \multirow[t]{6}{*}{ Blood system } & $\mathrm{CD}_{4}{ }^{+}$stem cell & + & - & $\mathrm{H}$ & $\mathrm{H}$ & $\mathrm{H}$ & $\mathrm{H}$ & $\mathrm{H}$ & $\mathrm{H}$ \\
\hline & 721 B lymphoblasts & + & - & $\mathrm{H}$ & $\mathrm{H}$ & $\mathrm{H}$ & $\mathrm{H}$ & M & $\mathrm{H}$ \\
\hline & CD19+B cell & + & - & $\mathrm{H}$ & $\mathrm{H}$ & $\mathrm{H}$ & $\mathrm{H}$ & M & $\mathrm{H}$ \\
\hline & Leukemia lymphoblastic & + & - & M & M & M & M & L & $L$ \\
\hline & Bonemarrow & + & - & $\mathrm{H}$ & M & $\mathrm{H}$ & $\mathrm{H}$ & M & $\mathrm{H}$ \\
\hline & Pituitariy & + & - & $\mathrm{H}$ & $\mathrm{H}$ & $\mathrm{H}$ & $\mathrm{H}$ & $\mathrm{H}$ & M \\
\hline \multirow[t]{7}{*}{ Head } & Prefrontal Cortex & + & - & $\mathrm{H}$ & $\mathrm{H}$ & $\mathrm{H}$ & $\mathrm{H}$ & $\mathrm{H}$ & $\mathrm{H}$ \\
\hline & Pineal & + & - & $\mathrm{H}$ & $\mathrm{H}$ & $\mathrm{H}$ & $\mathrm{H}$ & $\mathrm{H}$ & M \\
\hline & Tongue & + & - & $\mathrm{H}$ & M & $\mathrm{H}$ & $\mathrm{H}$ & L & M \\
\hline & Tonsil & + & - & $\mathrm{H}$ & M & M & $\mathrm{H}$ & L & L \\
\hline & Retina & + & - & $\mathrm{H}$ & $\mathrm{H}$ & $\mathrm{H}$ & $\mathrm{H}$ & $\mathrm{H}$ & M \\
\hline & Trigeminal ganglion & + & - & M & $\mathrm{H}$ & $\mathrm{H}$ & $\mathrm{H}$ & L & L \\
\hline & Cerebellum & + & - & $\mathrm{H}$ & $\mathrm{H}$ & M & $\mathrm{H}$ & $\mathrm{H}$ & M \\
\hline \multirow[t]{6}{*}{ Viscera } & Heart & + & - & $\mathrm{H}$ & $\mathrm{H}$ & $\mathrm{H}$ & $\mathrm{H}$ & $\mathrm{H}$ & M \\
\hline & Lung & + & - & $\mathrm{H}$ & $\mathrm{H}$ & $\mathrm{H}$ & $\mathrm{H}$ & $\mathrm{H}$ & M \\
\hline & Liver & + & - & $\mathrm{H}$ & $\mathrm{H}$ & $\mathrm{H}$ & $\mathrm{H}$ & $\mathrm{H}$ & M \\
\hline & Kidney & + & - & M & M & M & M & $\mathrm{H}$ & L \\
\hline & Smooth Muscles & + & - & $\mathrm{H}$ & $\mathrm{H}$ & $\mathrm{H}$ & $\mathrm{H}$ & $\mathrm{H}$ & M \\
\hline & Adipocyte & + & - & $\mathrm{H}$ & M & M & $\mathrm{H}$ & L & L \\
\hline \multirow[t]{2}{*}{ Secretory system } & Adrenalgland & + & - & M & M & $\mathrm{H}$ & $\mathrm{H}$ & L & L \\
\hline & Pancreaticlstet & + & - & $\mathrm{H}$ & $\mathrm{H}$ & $\mathrm{H}$ & $\mathrm{H}$ & $\mathrm{H}$ & M \\
\hline \multirow[t]{5}{*}{ Genital system } & Placenta & + & + & $\mathrm{H}$ & $\mathrm{H}$ & $\mathrm{H}$ & M & $\mathrm{H}$ & M \\
\hline & Fetalthyroid & + & + & $\mathrm{H}$ & M & M & M & $\mathrm{H}$ & M \\
\hline & Uterus & + & + & M & M & M & M & M & L \\
\hline & Testis & + & + & M & M & M & M & $\mathrm{H}$ & L \\
\hline & Ovary & + & + & M & M & L & L & $\mathrm{L}$ & M \\
\hline
\end{tabular}

domain [44]. E74A belongs to Ets transcription protein, which is involved in multifarious important biological processes. Study has demonstrated that ecdysone inducible TRFs E74A could directly regulate the EO gene expression in silkworm [45]. StuAp is a member of fungal TRFs family that regulates cell cycle progression or development. Further, StuAp belongs to a sub-family possessing the conserved APSES domain. Study has shown that StuAp acts as a transcriptional repressor in A.nidulans, but as a weak activator in budding yeast [46].

\section{The US12 transcription and ICP47 function during the HSV infection process}

To better understand the significance of the newly identified US12 transcriptional regulation, we summarize the US12 transcription and ICP47 function during the HSV infection process (Fig. 5). TAP plays a crucial role in MHC I antigen presentation and has become an important target for viral immune escape strategies. In the long-term process of virus-host co-evolution, herpes viruses independently obtained an efficient way to block TAP-mediated peptide transport via the viral immune evasion protein ICP47, which blocks the binding of peptide to TAP by capturing TAP in the endogenous conformation [27]. Interestingly, in our study, we found that two crucial TRFs, c-Rel and Oct-1, play a variety of roles in the growth, proliferation, and survival of mature $\mathrm{T}$ cells, which might associate with the viral immune evasion via HSV ICP47. Studies have shown that HSV-1 have evolved complex mechanisms to disrupt the antiviral response via affecting the NF- $\kappa B$. For example, in HSV-1, ICP0 interacts with p65 and p50 and then degrades p50 through regulating E3 ubiquitin ligase activity [47]. Protein kinase US3 was shown to inhibit NF-kB activity via making 665 hyperphosphorylation at serine 75 and blocking its nuclear translocation [48]. Besides, 


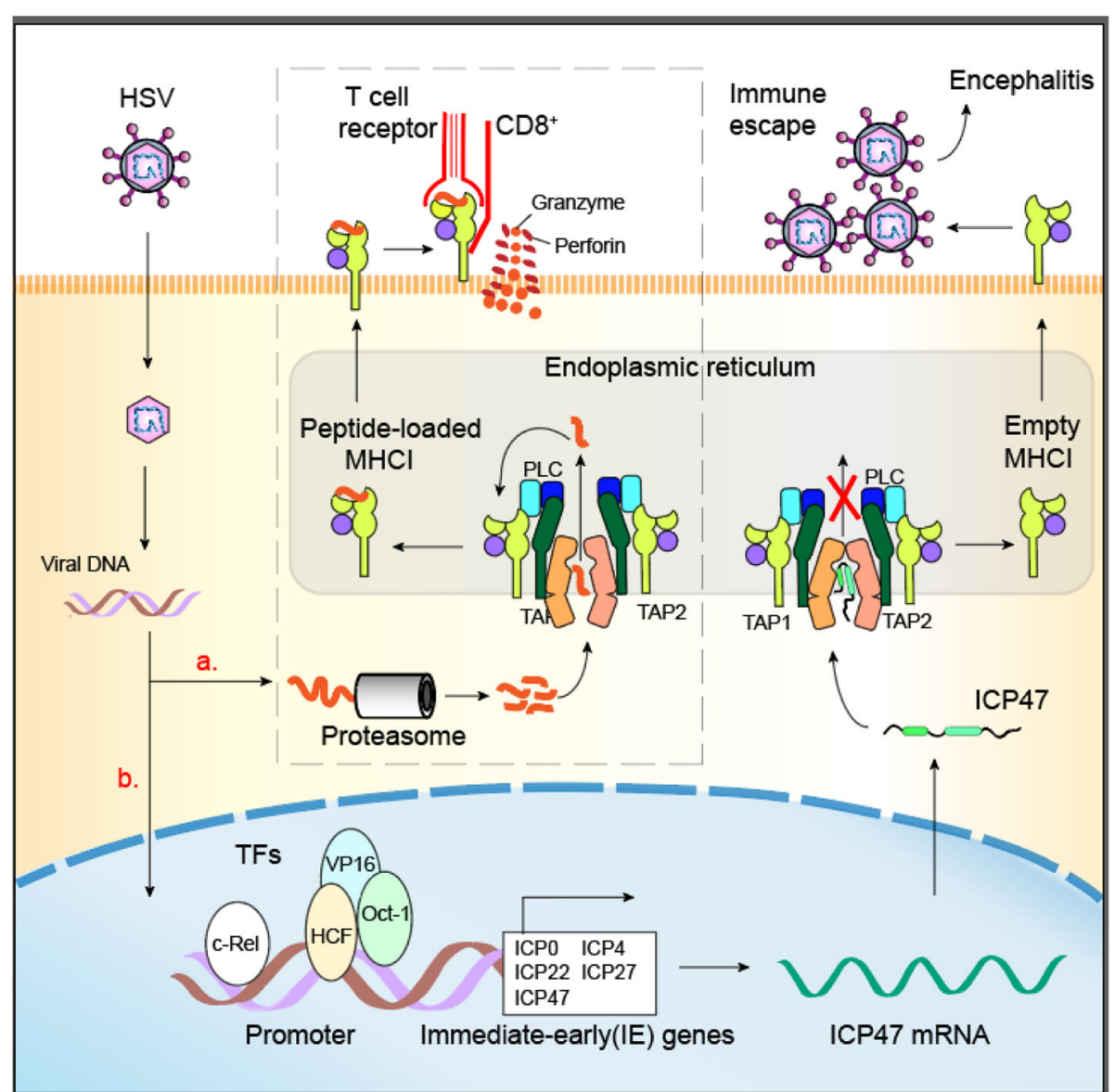

Fig. 5 ICP47 function during HSV infection. a Intracellular antigenic peptides, mainly generated by the proteasome, are transported into the endoplasmic reticulum by the TAP and then loaded onto the nascent MHC I molecules, which are exported to the cell surface and present peptides to the immune cells. Cytotoxic T lymphocytes recognize and kill the infected cells by granzyme and perforin. b ICP47 can preclude peptide binding and traps TAP in an inward-facing conformation. Binding of ICP47 stabilizes the inward-facing conformation, and thus prevents TAP from transitioning to an outward-facing state, resulting in the emergence of empty carrier MHC I molecules. Therefore, CD $8^{+} \mathrm{T}$ cells could not recognize them, and HSVs could avoid the immune responses. We hypothesize that c-Rel bind to its US12 TRS, and enhance US12 (ICP47) expression, leading to HSV-1 immune evasion and HSV-1 encephalitis

ICP27 blocks the phosphorylation of IкB to inhibit NF$\kappa \mathrm{B}$ activation. Furthermore, our data also shown that $\mathrm{c}-$ Rel is conserved in HSV-1, which inhibits NF-kB promoter activity. Importantly, Oct-1 plays a key role in CD4 $\mathrm{T}$ cells, mediating long-range chromosomal interactions and differentiation through regulating gene expression, and has a critical protection effect on viruses and pathogens [42] and further, Oct-1 also is conserved in $\mathrm{HSV}-2$.

\section{Discussion}

HSV ICP47 can bind TAP and block antigen presentation. Transcriptional regulation of US12 is important for ICP47 functioning. However, TRSs and TRFs of HSV US12 are seldom reported. In this study, we reported the transcriptional regulation region sequence of our newly isolated strain HSV-1-LXMW in China, and found it is closely related to HSV-1-CR38 and HSV-1-17 in UK. We identified eight different kinds of novel TRSs and TRFs of HSV US12 for the first time. These identified TRSs and TRFs are conserved among HSV-1 (c-Rel, Elk-1, Pax-4), HSV-2 (Oct-1, CF2-II, E74A, StuAp) or both of them (HNF-4). Two of the TRFs c-Rel and Oct-1 are biologically functional in vitro respectively in immune escape and viral replication during HSV infection. We further hypothesize a novel mechanism of HSV-1 encephalitis by c-Rel activated ICP47-mediated immune escape. These findings may have important implication to our understanding of HSV biology, infection, immunity and OVs.

oHSV-1 has become one of the most promising OVs at present [49]. In 2015, talimogene laherparepvec (TVEC), a kind of oHSV, was approved by FDA for the treatment of advanced melanoma [50-52]. In T-VEC, ICP47 was deleted to prevent limitation of viral antigen presentation, and increase the US11 gene expression, and virus replication in cancer cells without reducing 
tumor selectivity [53]. Considering that the immune escape function of ICP47, the construction of gene therapy vectors precede a new perspective. For instance, Adenoassociated virus gene therapy of Duchenne muscular dystrophy was achieved by expression ICP47 [54]. Additionally, study has also reported another recombinant adenovirus vector expressing ICP47 protein to reduce the stimulation of dendritic cells [55].

Future functional studies of these novel TRSs and TRFs, and their roles in HSV replication, infection, immunity, tissue tropism, encephalitis and OVs are warranted.

\section{Conclusions}

We identified eight different kinds of novel TRFs and TRFs of HSV US12 for the first time, and found they are conserved among HSV-1 (c-Rel, Elk-1, Pax-4), HSV-2 (Oct-1, CF2-II, E74A, StuAp) or both HSVs (HNF-4). The c-Rel and Oct-1 are biologically functional respectively in immune escape and viral replication during HSV infection. We further hypothesized a novel mechanism of HSV-1 encephalitis caused by c-Rel activated ICP47mediated immune escape.

\section{Supplementary information}

Supplementary information accompanies this paper at https://doi.org/10. 1186/s12985-020-01365-3.

Additional file 1. Using the nucleotide sequence database, we identified transcription regulatory regions of US12 (145851-148,050). The transcription regulatory regions are $2000 \mathrm{bp}$ upstream and $200 \mathrm{bp}$ downstream of US12 transcription initiation sites.

\section{Abbreviations}

HSV: Herpes simplex virus; ICP47: Infected cell polypeptide 47; oHSV: oncolytic HSV; TRSs: Transcription regulatory sequences; TRFs: Transcription regulatory factors; OVs: Oncolytic viruses; TK: Thymidine kinase; MHC-I: Major histocompatibility complex I; TAP: Transporter associated with antigen processing; NBDs: Nucleotide binding domains; ER: Endoplasmic reticulum; CTLs: Cytotoxic T lymphocytes; NF-kB: Nuclear factor KB; HNF-4: Hepatocyte nuclear factor 4; CF2-II: C(2)-H(2)-type zincfinger transcription factor II; T-VEC: Talimogene laherparepvec

\section{Acknowledgements}

None.

\section{Authors' contributions}

J.-T.C. and Y.-Y.W. designed the outline of the article and wrote it. Z.M. and H.-W.X designed the outline of the article, revised the initial draft and expanded the manuscript. L.-Z.Z. and B.-R.L. revised the manuscript. All made intellectual contributions. All authors approved the final manuscript.

\section{Funding}

This work was mainly supported by National Natural Science Foundation of China (81872412 to XHW, 81602303 to XY, 31700736 to WXW). We thank the Natural Science Foundation of Hubei Province (2019CFB591 to Z.M.), and Guangzhou Key Medical Discipline Construction Project (CSZ).

\section{Availability of data and materials}

All data from the current study are available from the corresponding author on request.
Ethics approval and consent to participate

Not applicable.

\section{Consent for publication}

Not applicable.

\section{Competing interests}

The authors have declared that no competing interest exists. Consent for publication.

\section{Author details}

${ }^{1}$ Laboratory of Oncology, School of Basic Medicine, Health Science Center, Yangtze University, 1 Nanhuan Road, Jingzhou 434023, Hubei, China. ${ }^{2}$ Department of Biochemistry and Molecular Biology, School of Basic Medicine, Health Science Center, Yangtze University, Jingzhou 434023, Hubei, China. ${ }^{3}$ Department of Interventional Therapy, Key laboratory of Carcinogenesis and Translational Research (Ministry of Education), Peking University Cancer Hospital \& Institute. 52, Fucheng Road, Haidian District, Beijing 100142, China. ${ }^{4}$ Department of Laboratory Medicine, School of Basic Medicine, Health Science Center, Yangtze University, 1 Nanhuan Road, Jingzhou 434023, Hubei, China. ${ }^{5}$ Department of Pathophysiology, School of Basic Medicine, Health Science Center, Yangtze University, Jingzhou 434023, Hubei, China. ${ }^{6}$ Department of Gastroenterology, The First Affiliated Hospital of Zhengzhou University, Zhengzhou 450052, China. ${ }^{7}$ State Key Laboratory of Respiratory Disease, Affiliated Cancer Hospital Institute of Guangzhou Medical University, Guangzhou 510095, China. 'Lianjiang People's Hospital, Guangdong 524400, China.

Received: 28 March 2020 Accepted: 23 June 2020

Published online: 10 July 2020

\section{References}

1. Ellison LM, Man Y, Stojadinovic A, Xin H, Avital I. Cytoreductive surgery and hyperthermic intraperitoneal chemotherapy in treatment of gastric cancer with peritoneal carcinomatosis. Chin J Cancer Res. 2017;29:86-92.

2. Liu Y, Yu C, Wu Y, Sun X, Su Q, You C, Xin H. CD44(+) fibroblasts increases breast cancer cell survival and drug resistance via IGF2BP3-CD44-IGF2 signalling. J Cell Mol Med. 2017;21:1979-88.

3. Xin HW, Ambe CM, Ray S, Kim BK, Koizumi T, Wiegand GW, Hari D, Mullinax JE, Jaiswal KR, Garfield SH, et al. Wnt and the cancer niche: paracrine interactions with gastrointestinal cancer cells undergoing asymmetric cell division. J Cancer. 2013;4:447-57.

4. Xin HW, Ambe CM, Hari DM, Wiegand GW, Miller TC, Chen JQ, Anderson AJ, Ray S, Mullinax JE, Koizumi T, et al. Label-retaining liver cancer cells are relatively resistant to sorafenib. Gut. 2013;62:1777-86.

5. Xin HW, Ambe CM, Miller TC, Chen JQ, Wiegand GW, Anderson AJ, Ray S, Mullinax JE, Hari DM, Koizumi T, et al. Liver label retaining Cancer cells are relatively resistant to the reported anti-Cancer stem cell drug metformin. J Cancer. 2016;7:1142-51.

6. Hari D, Xin HW, Jaiswal K, Wiegand G, Kim BK, Ambe C, Burka D, Koizumi T, Ray S, Garfield S, et al. Isolation of live label-retaining cells and cells undergoing asymmetric cell division via nonrandom chromosomal cosegregation from human cancers. Stem Cells Dev. 2011;20:1649-58.

7. Liu XQ, Xin HY, Lyu YN, Ma ZW, Peng XC, Xiang Y, Wang YY, Wu ZJ, Cheng $J T$, Ji JF, et al. Oncolytic herpes simplex virus tumor targeting and neutralization escape by engineering viral envelope glycoproteins. Drug Deliv. 2018;25:1950-62.

8. Russell SJ, Barber GN. Oncolytic viruses as antigen-agnostic Cancer vaccines. Cancer Cell. 2018;33:599-605

9. Lawler SE, Speranza MC, Cho CF, Chiocca EA. Oncolytic viruses in Cancer treatment: a review. JAMA Oncol. 2017;3:841-9.

10. Puzanov I, Milhem MM, Minor D, Hamid O, Li A, Chen L, Chastain M, Gorski KS, Anderson A, Chou J, et al. Talimogene Laherparepvec in combination with Ipilimumab in previously untreated, Unresectable stage IIIB-IV melanoma. J Clin Oncol. 2016:34:2619-26.

11. Poh A. First Oncolytic viral therapy for melanoma. Cancer Discov. 2016;6:6.

12. Agarwalla PK, Aghi MK. Oncolytic herpes simplex virus engineering and preparation. Methods Mol Biol. 2012;797:1-19.

13. Dai $X$, Zhou ZH. Structure of the herpes simplex virus 1 capsid with associated tegument protein complexes. Science. 2018;360. 
14. Johnston C, Koelle DM, Wald A. HSV-2: in pursuit of a vaccine. J Clin Invest. 2011:121:4600-9.

15. Duan J, Liuzzi M, Paris W, Liard F, Browne A, Dansereau N, Simoneau B, Faucher AM, Cordingley MG. Oral bioavailability and in vivo efficacy of the helicase-primase inhibitor BILS 45 BS against acyclovir-resistant herpes simplex virus type 1. Antimicrob Agents Chemother. 2003;47:1798-804.

16. Bernstock JD, Vicario N, Li R, Nan L, Totsch SK, Schlappi C, Gessler F, Han X, Parenti R, Beierle EA, et al. Safety and efficacy of oncolytic HSV-1 G207 inoculated into the cerebellum of mice. Cancer Gene Ther. 2019.

17. Russell SJ, Peng KW, Bell JC. Oncolytic virotherapy. Nat Biotechnol. 2012;30: 658-70.

18. Todo T, Martuza RL, Rabkin SD, Johnson PA. Oncolytic herpes simplex virus vector with enhanced MHC class I presentation and tumor cell killing. Proc Natl Acad Sci. 2001;98:6396-401.

19. Whitley RJ, Roizman B. Herpes simplex virus infections. Lancet. 2001;357: 1513-8.

20. Praest P, Luteijn RD, Brak-Boer IGJ, Lanfermeijer J, Hoelen H, ljgosse L, Costa Al, Gorham RD, Lebbink RJ, Wiertz EJHJ. The influence of TAP1 and TAP2 gene polymorphisms on TAP function and its inhibition by viral immune evasion proteins. Mol Immunol. 2018;101:55-64.

21. Oldham ML, Hite RK, Steffen AM, Damko E, Li Z, Walz T, Chen J. A mechanism of viral immune evasion revealed by cryo-EM analysis of the TAP transporter. Nature. 2016:529:537-40.

22. Matschulla T, Berry R, Gerke C, Döring M, Busch J, Paijo J, Kalinke U, Momburg F, Hengel $\mathrm{H}$, Halenius A. A highly conserved sequence of the viral TAP inhibitor ICP47 is required for freezing of the peptide transport cycle. Sci Rep. 2017;7.

23. Liu R, Varghese S, Rabkin SD. Oncolytic herpes simplex virus vector therapy of breast cancer in C3(1)/SV40 T-antigen transgenic mice. Cancer Res. 2005; 65:1532-40.

24. Messerli SM, Prabhakar S, Tang Y, Mahmood U, Giovannini M, Weissleder R, Bronson R, Martuza R, Rabkin S, Breakefield XO. Treatment of schwannomas with an oncolytic recombinant herpes simplex virus in murine models of neurofibromatosis type 2. Hum Gene Ther. 2006;17:20-30.

25. Fukuhara H, Martuza RL, Rabkin SD, Ito Y, Todo T. Oncolytic herpes simplex virus vector g47delta in combination with androgen ablation for the treatment of human prostate adenocarcinoma. Clin Cancer Res. 2005;11: 7886-90.

26. Raafat N, Sadowski-Cron C, Mengus C, Heberer M, Spagnoli GC, Zajac P. Preventing vaccinia virus class-I epitopes presentation by HSV-ICP47 enhances the immunogenicity of a TAP-independent cancer vaccine epitope. Int J Cancer. 2012;131:E659-69.

27. Scaria A, Sullivan JA, St. George JA, Kaplan JM, Lukason MJ, Morris JE, Plog M, Nicolette C, Gregory RJ, Wadsworth SC. Adenoviral vector expressing ICP47 inhibits adenovirus-specific cytotoxic T lymphocytes in nonhuman primates. Mol Ther. 2000;2:505-14.

28. S O, FJ H, JR D, A H, S M, WS L, SJ T: Phosphorylation of the VP16 transcriptional activator protein during herpes simplex virus infection and mutational analysis of putative phosphorylation sites. Virology 2006, 345: 468-481.

29. Suk $H$, Knipe DM. Proteomic analysis of the herpes simplex virus 1 virion protein 16 transactivator protein in infected cells. Proteomics. 2015;15:1957-67.

30. Wang YY, Lyu YN, Xin HY, Cheng JT, Liu XQ, Wang XW, Peng XC, Xiang Y, Xin WW, Lu CB, et al. Identification of putative UL54 (ICP27) transcription regulatory sequences binding to Oct-1, v-Myb, Pax-6 and hairy in herpes simplex viruses. J Cancer. 2019;10:430-40.

31. Mancini M, Caignard G, Charbonneau B, Dumaine A, Wu N, Leiva-Torres GA, Gerondakis S, Pearson A, Qureshi ST, Sladek R, Vidal SM. Rel-dependent immune and central nervous system mechanisms control viral replication and inflammation during mouse herpes simplex encephalitis. J Immunol. 2019;202:1479-93.

32. SL S, J H, PM P: Differential regulation of the HIV-1 LTR by specific NF-kappa B subunits in HSV-1-infected cells. Virology 1996, 224:214-223.

33. Nogueira ML, Wang VE, Tantin D, Sharp PA, Kristie TM. Herpes simplex virus infections are arrested in Oct-1-deficient cells. Proc Natl Acad Sci U S A. 2004;101:1473-8.

34. Akhova $\mathrm{O}$, Bainbridge $M$, Misra $V$. The neuronal host cell factor-binding protein Zhangfei inhibits herpes simplex virus replication. J Virol. 2005;79: 14708-18.
35. V M, S W, P Y, S H, P OH: Conformational alteration of Oct-1 upon DNA binding dictates selectivity in differential interactions with related transcriptional coactivators. Mol Cell Biol 1996, 16:4404-4413.

36. Advani SJ, Durand LO, Weichselbaum RR, Roizman B. Oct-1 is posttranslationally modified and exhibits reduced capacity to bind cognate sites at late times after infection with herpes simplex virus 1. J Virol. 2003;77: 11927-32.

37. RF G, P OH: Sequence, function, and regulation of the Vmw65 gene of herpes simplex virus type 2. J Virol 1991, 65:6705-6713.

38. Mancini M, Caignard G, Charbonneau B, Dumaine A, Wu N: Rel-Dependent Immune and Central Nervous System Mechanisms Control Viral Replication and Inflammation during Mouse Herpes Simplex Encephalitis 2019, 202: 1479-1493.

39. Gilmore TD, Gerondakis S. The c-Rel transcription factor in development and disease. Genes Cancer. 2011:2:695-711.

40. Ducker C, Chow LKY, Saxton J, Handwerger J, McGregor A, Strahl T, Layfield $\mathrm{R}$, Shaw PE. De-ubiquitination of ELK-1 by USP17 potentiates mitogenic gene expression and cell proliferation. Nucleic Acids Res. 2019;47:4495-508.

41. Sakata N, Yoshimatsu G, Kodama S. Development and characteristics of pancreatic epsilon cells. Int J Mol Sci. 2019;20.

42. Hwang SS, Kim LK, Lee GR, Flavell RA. Role of OCT-1 and partner proteins in T cell differentiation. Biochim Biophys Acta. 2016;1859:825-31.

43. Tsukamoto D, Ito M, Takamatsu N. HNF-4 participates in the hibernationassociated transcriptional regulation of the chipmunk hibernation-related protein gene. Sci Rep. 2017;7:44279.

44. Gogos JA, Hsu T, Bolton J, Kafatos FC. Sequence discrimination by alternatively spliced isoforms of a DNA binding zinc finger domain. Science. 1992;257:1951-5.

45. Sun W, Wang CF, Zhang Z. Transcription factor E74A affects the ecdysone titer by regulating the expression of the EO gene in the silkworm, Bomby mori. Biochim Biophys Acta Gen Subj. 2017;1861:551-8.

46. Zhao $Y$, Su H, Zhou J, Feng H, Zhang KQ, Yang J. The APSES family proteins in fungi: characterizations, evolution and functions. Fungal Genet Biol. 2015; $81: 271-80$.

47. Zhang J, Wang K, Wang S, Zheng C. Herpes simplex virus 1 E3 ubiquitin ligase ICPO protein inhibits tumor necrosis factor alpha-induced NF-kappaB activation by interacting with p65/RelA and p50/NF-kappaB1. J Virol. 2013; 87:12935-48.

48. Wang $\mathrm{K}$, Ni L, Wang S, Zheng C. Herpes simplex virus 1 protein kinase US3 hyperphosphorylates p65/RelA and dampens NF-kappaB activation. J Virol. 2014:88:7941-51.

49. Wang D, Wang XW, Peng XC, Xiang Y, Song SB, Wang YY, Chen L, Xin WW, Lyu $Y N$, Ji J, et al. CRISPR/Cas9 genome editing technology significantly accelerated herpes simplex virus research. Cancer Gene Ther. 2018;25:93-105.

50. Killock D. Skin cancer: T-VEC oncolytic viral therapy shows promise in melanoma. Nat Rev Clin Oncol. 2015;12:438.

51. Johnson DB, Puzanov I, Kelley MC. Talimogene laherparepvec (T-VEC) for the treatment of advanced melanoma. Immunotherapy. 2015;7:611-9.

52. Andtbacka R, Collichio F, Amatruda T, Senzer N, Chesney J, Delman K, Spitler L, Puzanov I, Doleman S, Ye Y, et al. OPTiM: a multicenter, randomized phase 3 trial of Talimogene Laherparepvec versus subcutaneous GM-CSF for the treatment of Unresected stage IIIB/C and IV melanoma. J der Deutschen Dermatologischen Gesellschaft. 2013;11:8-8.

53. Grigg C, Blake Z, Gartrell R, Sacher A, Taback B, Saenger Y. Talimogene laherparepvec $(\mathrm{T}-\mathrm{Vec})$ for the treatment of melanoma and other cancers. Semin Oncol. 2016:43:638-46.

54. Shao W, Chen X, Samulski RJ, Hirsch ML, Li C. Inhibition of antigen presentation during AAV gene therapy using virus peptides. Hum Mol Genet. 2018;27:601-13.

55. Wang P, Kan Q, Yu Z, Li L, Zhang Z, Pan X, Feng T. Recombinant adenovirus expressing ICP47 gene suppresses the ability of dendritic cells by restricting specific T cell responses. Cell Immunol. 2013;282:129-35.

\section{Publisher's Note}

Springer Nature remains neutral with regard to jurisdictional claims in published maps and institutional affiliations. 